\title{
The Correlation of Vibration Signal Features in Grinding of Advanced Ceramics
}

\author{
P. O. Conceição Junior, M. Marchi, P. R. Aguiar, E. C. Bianchi and T. V. França
}

\begin{abstract}
Monitoring the grinding of ceramics using the vibration signal has been presented as an alternative for the diagnosis of the workpiece surface. This paper has the objective of studying the vibration signal through spectral analysis in order to monitor the grinding process, and looking for the best parameters that could be related to the surface integrity in the finished workpieces of ceramics. Thus, grinding tests were carried out on alumina ceramic specimens in different depths of cut. The workpieces were evaluated after grinding process by measuring the surface roughness $\mathrm{Ra}$ and confocal microscopy. For monitoring was used an accelerometer and vibration signal was collected by an oscilloscope. Digital signal processing techniques were performed, identifying a range of frequencies between $800 \mathrm{~Hz}$ and $2 \mathrm{kHz}$ that best correlate with the condition of the machined ceramic. There was a correlation between the vibration and the integrity of the ceramics workpiece after grinding process. Moreover, the increase of the vibration is directly proportional to the surface roughness each cutting depth used. It follows that the vibration can be used to monitor the grinding of ceramics due to their relationship with the condition of the workpieces.
\end{abstract}

Keywords - Monitoring of Grinding Process, Advanced Ceramics, Vibration Signal, Spectral Analysis.

\section{INTRODUÇÃO}

A UTILIZAÇÃO de materiais cerâmicos para componentes estruturais está evoluindo de uma curiosidade acadêmica a realidade prática. $\mathrm{O}$ crescente interesse em cerâmica pode ser creditado para o desenvolvimento de materiais com melhores propriedades, que possuam uma maior resistência à temperatura ambiente e temperaturas elevadas, maior tenacidade à fratura, e propriedades mais uniformes. Para obter a forma desejada na usinagem destes materiais é necessário o uso do processo de retificação. A viabilidade econômica do alto desempenho da cerâmica depende de quão eficientemente elas podem ser usinadas pela retificação [1].

De todos os processos de usinagem de uso comum, a retificação é o mais caro por unidade de volume de remoção de material. Na fabricação de componentes cerâmicos, a retificação pode compreender até $80 \%$ do custo total [2]. A

P. O. Conceição Junior, Departamento de Engenharia Elétrica da Faculdade de Engenharia de Bauru, UNESP, Bauru SP, Brasil, pedrojunior5@aedu.com

M. Marchi, Departamento de Engenharia Mecânica da Faculdade de Engenharia de Bauru, UNESP, Bauru SP, Brasil, marcelo.marchi@outlook.com

P. R. Aguiar, Departamento de Engenharia Elétrica da Faculdade de Engenharia de Bauru, UNESP, Bauru SP, Brasil, aguiarpr@feb.unesp.br

E.C. Bianchi, Departamento de Engenharia Mecânica da Faculdade de Engenharia de Bauru, UNESP, Bauru SP, Brasil, bianchi@feb.unesp.br

T.V. França, Departamento de Engenharia Mecânica da Faculdade de Engenharia de Bauru, UNESP, Bauru SP, Brasil, thiagovf@feb.unesp.br retificação eficiente de alto desempenho da cerâmica requer a seleção dos parâmetros de operação para maximizar taxa de remoção enquanto controla a integridade superficial. Reduzir custos de retificação usando taxas de remoção mais rápidas é um recurso limitado, principalmente por danificar a superfície da peça de cerâmica, levando a sua degradação $[3,4]$.

As características da retificação cerâmicas avançadas são muito diferentes das características dos metais. Portanto, é necessário efetuar outros estudos para alcançar uma compreensão mais abrangente e um melhor controle dos parâmetros, a fim de obter um bom acabamento superficial. Algumas dificuldades encontradas na usinagem são a elevada dureza e rigidez das cerâmicas, por exemplo, [5].

Por outro lado, a rugosidade que também é influenciada pela retificação na cerâmica, tem se tornado um fator importante para avaliar a qualidade da peça usinada. No final do processo, o acabamento da superfície afeta as características da peça, como a resistência à fadiga, fratura, assim como a rugosidade [6]. Apesar da importância de uma avaliação microscópica da superfície das peças, a rugosidade é medida, em muitos casos, utilizando métodos tradicionais e com a haste de perfilometria óptica. Esta aquisição contínua torna-se inviável numa linha de produção, devido à parada constante da máquina para medir a rugosidade $[7,8]$.

Outro fator importante é a qualidade estrutural da cerâmica. Como as cerâmicas são frágeis, a falha mecânica geralmente ocorre pela propagação de trincas. Uma análise das cerâmicas após a usinagem, por meio de métodos de microscopia, por exemplo, é extremamente importante para qualificar danos superficiais nas peças. Esta qualificação é extremamente necessária, porque a qualidade superficial das cerâmicas avançadas pode ser aprimorada se a configuração do processo de retificação for realizada de maneira correta [9].

Uma das formas de aprimorar o processo é estimar a rugosidade e estudar a topografia das cerâmicas após a usinagem, a partir do monitoramento do processo de retificação. A retificação automática e inteligente é utilizada por indústrias para a produção com alta qualidade de acabamento e geometria [6]. Métodos indiretos de monitoramento desse processo são utilizados para avaliar a qualidade superficial e estrutural das peças usinadas. $\mathrm{O}$ processo envolve a coleta, processamento e análise de dados relacionados com a peça usinada em várias condições experimentais e interpretação dos resultados para as aplicações em situações reais [10], e contam com alguns sinais sensoriais, tais como forças, energia, vibração e emissão acústica que se correlacionam com a condição de ferramenta [10,11 e 12]. 
De acordo com Zeng \& Forssberg [14], o processo de retificação emite fortes sinais de vibração que se apresentam na forma de variações de pressão acustica e vibrações mecânicas. O sinal de vibração medido pode ser estudado utilizando métodos de análise no domínio do tempo, estimação espectral e suas variações podem ser regidas de acordo com as características processo de retificação. [14, 15].

Assim, considerando a importância de se avaliar o comportamento de peças cerâmicas usinadas, no presente trabalho se investigará o sinal de vibração no domínio do tempo e seu conteúdo em frequência por meio da estimação espectral, buscando a extração das características que melhor representem a rugosidade e a ocorrência de trincas nas peças retificadas. A justificativa de se utilizar esse método parte da hipótese de que, empregando a estimação espectral no tratamento de sinais é possível identificar as melhores faixas de frequência, que possivelmente se correlacionam com as condições do processo, que no caso é a retificação de cerâmicas. A estimação espectral é método de análise do domínio de frequência usado para prever um possível padrão de comportamento de sinais aleatórios, tais como os sinais de vibração emitido de um processo retificação. Com o presente estudo, um sistema de monitoramento pode ser proposto para permitir a melhor seleção e configuração dos parâmetros da retificação de cerâmicas, contribuindo para a automatização do processo.

\section{CARACTERIZAÇÃO DE CERÂMICAS RETIFICADAS}

As cerâmicas avançadas, que são as cerâmicas de qualidade elevada, desenvolvidas com matérias-primas sintéticas de alta pureza e através de processos rigorosos, tais como sinterização e prensagem, $\mathrm{ABC}$ apud [16], tem ganhado importância nos últimos anos, devido seu alto desempenho, suas boas propriedades, como dureza, resistência à temperatura e ao desgaste. Estes componentes estão sendo cada vez mais aplicados em indústrias de manufatura em geral [17]. No entanto, a utilização efetiva da cerâmica avançada é limitada em alguns aspectos, principalmente por causa do custo elevado do processamento destes materiais por retificação quando comparado com o aço, por exemplo, para garantir simultaneamente a qualidade da peça [18].

O processo de retificação é a etapa mais importante na usinagem de cerâmicas avançadas, é altamente complexo e envolve o contato entre um grande número de partículas abrasivas, com a superfície da peça [19]. Há uma variedade de técnicas de caracterização superficial e subsuperficial de peças manufaturadas por meio dos finos processos de acabamento. A rugosidade destaca-se como um dos mais usados parâmetros de qualificação da topografia de superfícies retificadas, a qual é representada pelo valor médio aritmético, $R_{a}$, o valor médio quadrático, $R_{q}$, e o valor entre o pico e vale, $R_{t}$ [20]. Outras técnicas utilizadas para a caracterização superficial e subsuperficial da cerâmica usinada tem sido encontradas na literatura, tais como, as ópticas que incluem métodos que medem a transmissão ou reflexão da luz incidente, dentre elas a microscopia confocal, por exemplo [22,23,24]. Técnicas como essas são utilizadas para avaliar a qualidade superficial e estrutural das cerâmicas usinadas, encontrar possíveis danos, como fissuras ou trincas. [25,27].

\section{MONITORAMENTO DO PROCESSO DE RETIFICAÇÃO}

Samhouri e Surgenor [6], afirmam que o monitoramento do processo de retificação serve para detectar problemas, fornecer informações para o aprimoramento e controle do processo e, por último, contribuir para o estabelecimento de parâmetros que são necessários para determinar as melhores condições de funcionamento. Além disso, os avanços tecnológicos na produção de materiais cerâmicos tornaram possível aplicar técnicas que foram anteriormente utilizados com metais.

De acordo com Agarwal \& Rao [5], a análise direta da superfície da peça exige que o funcionamento da máquina seja interrompido, e a extração da ferramenta e peça visualmente inspecionadas, o que resulta em tempos de paragem e custos intervenção do utilizador humano. Métodos indiretos de análise, no entanto, permite um monitoramento sem desligar a máquina e interromper o processo. Os métodos indiretos dependem da relação entre as condições de ferramentas e sinais mensuráveis de sensores (tais como forças, energia, vibração e emissão acústica) para detecção de condições da ferramenta e têm sido extensivamente estudados [26]. O foco deste trabalho é utilizar o sinal de vibração no monitoramento do processo de retificação de cerâmicas.

No processo de retificação ocorrem tanto vibrações forçadas como as autoexcitadas. Vibrações forçadas são causadas pela rotação de eixos desbalanceados, colisões entre elementos no final dos seus movimentos, etc. Vibrações autoexcitadas são causadas por colisões entre grãos abrasivos e partículas duras da peça, espessura de cavaco variável, atrito e mecanismo da formação de cavaco [11]. Acelerômetros são usados para medir vibrações em monitoramento de processos de usinagem. Entretanto, o uso de sinais de vibração no monitoramento do processo de retificação ainda é raro.

Pesquisas foram realizadas para correlacionar os sinais de vibração com características do processo de usinagem. Por exemplo, Yamamoto et al., apud [27] monitorou a vibração do rebolo (fixando um sensor sobre o rolamento do rebolo) para detectar o entupimento dos poros do rebolo por cavaco. Tendo em vista a este objetivo, eles utilizaram filtros digitais adaptativos e criou um índice com base nos resultados destes filtros, chamado o índice de o padrão do sinal. Este índice mostrou ter uma relação direta e satisfatória com o volume de cavaco acumulado no rebolo.

Os sinais de vibração podem fornecer informações variadas sobre o processo de retificação. Porém, análises mais rigorosas necessitam de processamento desse sinal para obtenção de informações correlacionando o sinal e os fenômenos sob estudo, tais como alta rugosidade ou danos térmicos e mecânicos nas peças, por exemplo, que são variações capitadas pelo sensor. Entre as estatísticas para a monitoramentode sinais, a mais usada é o RMS (do inglês Root Mean Square ou valor médio quadrático), expresso da seguinte maneira em (1), de acordo com [28]: 
$x_{R M S}=\sqrt{\frac{1}{T} \int_{t_{0}}^{t+T} x^{2}(t) \cdot d t}$

Hassui et al. [29] utilizou o sinal de vibração para a avaliação do processo de retificação cilíndrica de mergulho de peças de aço ABNT 52.100 . O sinal de vibração do processo foi utilizado com sucesso na determinação indireta do melhor momento para a dressagem do rebolo. Foi verificado ainda que a vibração seja capaz de indicar com clareza as três fases da retificação cilíndrica externa de mergulho: deformação elástica inicial, remoção de material e spark-out.

Em um estudo David \& Warneck [30] apud Hassui et al. [11] relacionaram o sinal de vibração com as forças dinâmicas da retificação. A pesquisa mostrou que estas forças fazem o sistema (a máquina o rebolo e a peça) vibrar e que é possível captar o aumento de amplitude da vibração em algumas frequências à medida que o rebolo perde a afiação. Os autores concluíram que as vibrações irregulares de pequenas amplitudes são típicas do processo sem a ocorrência de trepidação, já quando ocorrem, as vibrações autoexcitadas são praticamente harmônicas e de altas amplitudes.

$\mathrm{Na}$ pesquisa de Chuangwen et al., [31] foi usado um método de avaliação da condição de desgaste de uma ferramenta através de redes neurais e análise de pacotes wavelet por meio do sinal de vibração. $\mathrm{O}$ método apresentou uma análise de bandas de frequências para o monitoramento do desgaste da ferramenta, podendo ser usado para estimar sua condição durante o uso com boa precisão.

A análise espectral é um método eficiente a ser aplicado no estudo do sinal de vibração. Baseia-se na seleção de faixas de frequências que irão caracterizar a variável física que é desejável controlar ou estimar, ou seja, as faixas de frequência em que o sinal apresenta correlação com o processo real que esta sendo observado mediante aquele sinal [14]. Um dos métodos utilizados na análise de frequência de sinal é a transformada rápida de Fourier (FFT). A estimação espectral de processos discretamente amostrados, geralmente é baseada em procedimentos que empregam a FFT. Esta abordagem é computacionalmente eficiente e produz resultados satisfatórios no processamento digital de sinais.

Com base na FFT, outros métodos de estimação espectral são propostos, uma vez que sinais provenientes do processo de retificação, por exemplo, traduzem a natureza estocástica do processo. Desse modo, para prever um padrão de comportamento de um sinal de natureza aleatória, são necessárias ferramentas de estimação [32]. Nesse sentido, os estudos caminharam para a estimação por meio da densidade espectral de potência (PSD) de um sinal, que é um método evoluído da estimação espectral, que mostra justamente como a potência do sinal está distribuída ao longo de todas as suas componentes de frequência. A PSD retorna a energia total presente no sinal, a partir de um deslocamento entre amostras, uma sequência de autocorrelação entre sinais diversos ou dentro do próprio sinal obtida de uma sequência de valores aleatórios para detectar padrões em sinais. A estimação espectral clássica consiste nos métodos de obtenção da estimativa da PSD [32], [33]. O mais simples é conhecido como método de "periodograma". O método Welch é uma versão melhorada do periodograma, usado para determinar a densidade de energia dos componentes de frequência num sinal baseado na DFT [34]. O PSD de um sinal pelo método Welch pode ser obtido por meio da divisão do sinal em segmentos. Calcula-se o espectro de potência de cada segmento usando a FFT e, em seguida, as médias dos espectros [35].

$\mathrm{O}$ processo de retificação pode causar dano às peças e, em muitos casos esses danos não poderiam ser detectados através de métodos que utilizam sinais de excitação em baixas frequências devido a essas frequências serem provenientes de rudios ou frequências naturais da própria máquina. Um dos índices mais empregados em sistemas de Monitoramento de Integridade Estrutural (SHM), que é um processo de detecção de danos, por conseguinte definido como o reconhecimento e monitoramento de uma alteração adversa causada na estrutura que afeta o desempenho do sistema, esse índice é o RMSD (Root Mean Square Deviation) de acordo com [36], como apresentado na Equação (2):

$R M S D=\sum_{k=\omega_{l}}^{\omega F} \sqrt{\frac{\left[X_{E, D}(k)-X_{E, H}(k)\right]^{2}}{X_{E, H}^{2}(k)}}$

Onde $Z_{E, D}$ e $Z_{E, H}$ representam os sinais correspondentes à estrutura danificada e íntegra, para a estrutura sem dano e com dano, medidas na frequência $\mathrm{k}$ que varia de $\omega_{l}$ (a frequência inicial) até $\omega_{F}$ (a frequência final), respectivamente. Outro índice estatístico bastante utilizado em SHM é o CCDM (Correlation Coefficient Deviation Metric) de acordo com (Marqui et al. 2008), dado pela Equação (3).

$C C D M=1-\frac{\sum_{k=\omega_{l}}^{\omega F}\left[Z_{E, H}(k)-\bar{Z}_{E, H}\right]\left[Z_{E, D}(k)-\bar{Z}_{E, D}\right]}{\sqrt{\sum_{k=\omega_{l}}^{\omega F}\left[Z_{E, H}(k)-\bar{Z}_{E, H}\right]^{2}} \sqrt{\sum_{k=\omega_{l}}^{\omega F}\left[Z_{E, D}(k)-\bar{Z}_{E, D}\right]^{2}}}$

Numa etapa subsequente, métricas estatísticas são determinadas a partir dos sinais de impedância para detecção de danos estruturais.

\section{MÉTODOLOGIA EXPERIMENTAL}

Um banco de ensaios foi projetado no laboratório de usinagem por abrasão (LUA). Foi utilizada uma retificadora tangecial plana modelo RAPH-1055 (Sulmecânica, Brasil), equipada com um rebolo de diamante sintético (especificações: SD 126 MN 50 B2). A operação de dressagem foi realizada com um dressador de ponta unica diamantado tipo cluster no inicio e final dos ensaios As peças do ensaio consistiam em barras rectangulares de ceramica alumina comercial, compostas por $96 \%$ de óxido de alumínio e $4 \%$ de óxidos de fluxo, tais como $\mathrm{SiO} 2, \mathrm{CaO}$ e $\mathrm{MgO}$, as quais foram produzidas por prensagem uniaxial e sinterização com temperatura de $1600^{\circ} \mathrm{C}$. A Tabela 1 lista os parâmetros ajustados ao sistema. 
TABELA 1

CONFIGURAÇÃO DOS PARÂMETROS DE RETIFICAÇÃO

\begin{tabular}{|l|c|}
\hline Velocidade de Corte $\left(v_{s}\right)$ & $35 \mathrm{~m} / \mathrm{s}$ \\
\hline Velocidade da Peça $\left(v_{w}\right)$ & $58 \mathrm{~mm} / \mathrm{s}$ \\
\hline Vazão do Fluido & $27.51 \mathrm{I} / \mathrm{min}$. \\
\hline Pressão do Fluido & $<0.2 \mathrm{Kgf} / \mathrm{cm}^{2}$ \\
\hline Concentração do Fluido & $2.5 \%$ em óleo \\
\hline Especificação do Fluido & Shell DMS $3200 \mathrm{~F}-1$ \\
\hline Emulsão & Água \\
\hline Tipo de óleo & Solúvel, semi-sintético \\
\hline Profundidades de corte $(\mu \mathrm{m})$ & $25,50,105,150,210$ e 350 \\
\hline
\end{tabular}

As dimensões eram de $20 \times 20 \times 7 \mathrm{~mm}$ ( $\mathrm{C} \times \mathrm{L} \times \mathrm{A})$. O material das amostras foi caracterizado no Instituto de Pesquisas Tecnológicas e apresentou densidade aparente de $3,701 \pm 0,002 \mathrm{~g} / \mathrm{cm} 3$. A amostras possuem tenacidade à Fratura de 3,18 $\pm 0,07 \mathrm{MPa} . \mathrm{m} 1 / 2$, de acordo com a norma ASTM C1421-01. Uma microdureza Vickers de $1339 \pm 47$ HV1 (norma JIS R1610-1991) e o tamanho médio de grão de $1,57 \mu \mathrm{m}$ (norma ASTM E1382-97)

Os ensaios foram realizados em 6 profundidades de corte listadas na Tabela 1. Cada um dos testes do experimento foi constituído de passadas do rebolo sobre o corpo de prova de cerâmica nas profundidades de corte definidas, sendo uma profundidade para cada corpo de prova, e o sinal de vibração coletado em cada uma das passadas. A razão de variar a profundidade de corte é o fato de se obter diferentes espessuras de material removido da peça, ampliando os resultados desse método para diferentes condições de corte.

Para a medição da rugosidade foi utilizado um rogosímetro portátil digital, modelo Surtronic 3+, fabricadas pela Taylor Robson. As medições foram realizadas na direção do avanço, em quatro pontos ao longo de cada peça usinada, adotando o parâmetro $R_{a}$. A análise superficial sem contato ocorreu no microscópio confocal da marca Leica, modelo DCM 3D, com aplicação de 800x, operado pelo programa Leica Scan. Esta análise visou observar possíveis trincas induzidas durante a retificação.

A aquisição da vibração foi feita por um acelerômetro da empresa PCB Piezotronics, consistindo de um sensor fixo ao suporte da peça e um módulo amplificador com ganho utilizado de 20. As especificações do acelerômetro são: sensibilidade a $100 \mathrm{~Hz}$ de $10 \mathrm{mV} / \mathrm{g}$, sensibilidade transversal de $8 \%$, tempo de descarga constante de 8 segundos, frequência de ressonância de $55.4 \mathrm{kHz}$ e resposta em frequência de até 10 $\mathrm{kHz}$. Os sinais foram coletados por um osciloscópio, modelo DL850, da empresa Yokogawa, com frequência de amostragem de $2 \mathrm{MHz}$.

O processamento digital de sinais foi realizado de acordo com a Fig. 1. Inicialmente foi aplicado no sinal puro de vibração, com o uso do MATLAB, um filtro digital butterworth, passa-baixa de quinta ordem, cuja frequência de corte foi $10 \mathrm{kHz}$. O sinal de vibração é filtrado de acordo com as características do acelerômetro usado. Nesse caso o fabricante recomenda até $10 \mathrm{kHz}$. Em seguida, foi realizada a análise espectral, a fim de encontrar a melhor faixa de frequência que pode estar associada ao comportamento mecânico do processo. Neste caso, foi obtido a FFT e o PSD do sinal, com janela de Hanning considerando 8192 amostras. O PSD foi obtido por meio do método Welch, implementando a função pwelch no MATLAB.
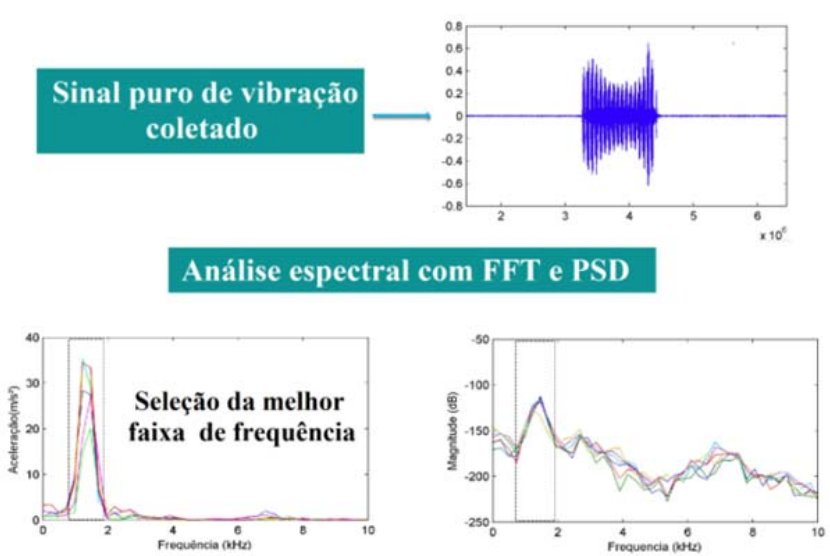

Cálculo do RMS

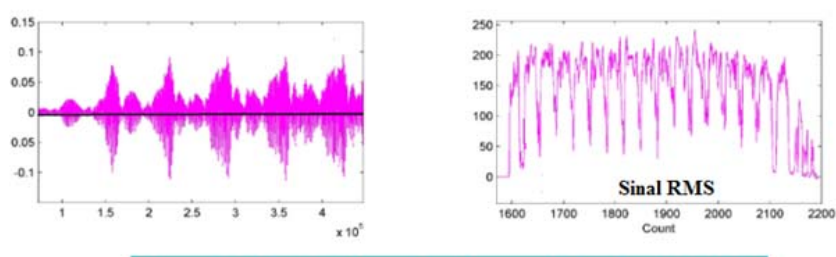

Geração dos índices de danos RMSD e CCDM

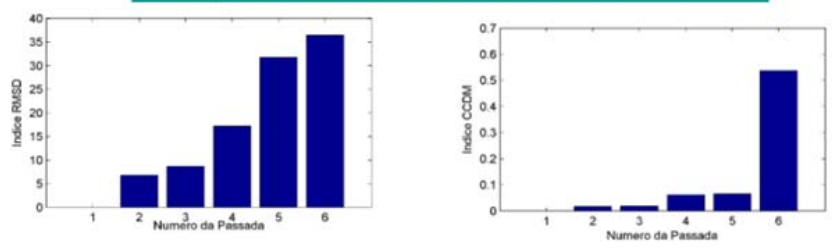

Figura 1. Etapas de processamento digital do sinal de vibração.

De acordo com a Fig. 1, foi aplicado no sinal puro um filtro digital na faixa de frequência selecionada, a partir desse sinal foi obtido o valor RMS em blocos de 2048 amostras do sinal, o que equivale $1 \mathrm{~ms}$. No sentido de encontrar um relacionamento entre o sinal de vibração e danos superficiais causados pela retificação no corpo de prova de cerâmica usinado, foram gerados os índices de danos RMSD e CCDM, tendo como referência a primeira peça.

\section{AVALIAÇÃO DA QUALIDADE SUPERFICIAL E ESTRTURAL DAS CERÂMICAS.}

A seguir são apresentados os resultados obtidos no experimento, quanto à caracterização das cerâmicas após a retificação. Inicialmente serão apresentados os resultados dos ensaios de microscopia confocal na Fig. 2, o qual foi realizado em uma região de interesse da superficie das peças. A análise foi feita para cada pronfudidades de corte, tendo como objetivo detectar microdefeitos na superficie de alumina retificada. A partir de observações realizadas nas imagens da Fig. 2, os resultados para as cerâmicas usinadas em condições de corte mais brandas não demostraram efeitos significativos da retificação em suas superfícies, como por exemplo, nas profundidades de corte de $25 \mu \mathrm{m}$ e $50 \mu \mathrm{m}$. 


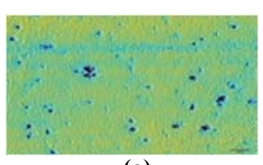

(a)

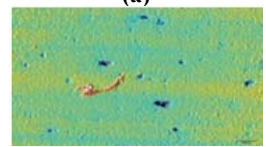

(d)

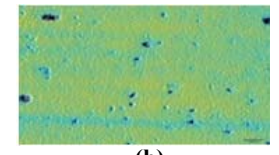

(b)

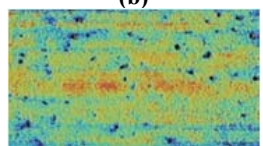

(e)

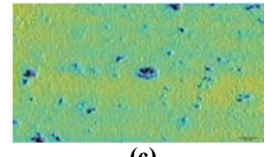

(c)

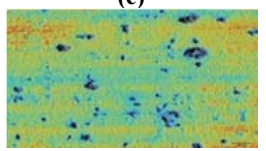

(f)
Figura 2. Superficie da cerâmica após a retificação, obtida por microscopia confocal; (a) $25 \mu \mathrm{m}$; (b) $50 \mu \mathrm{m}$; (c) $105 \mu \mathrm{m}$; (d) $150 \mu \mathrm{m}$; (e) $210 \mu \mathrm{m}$ e (d) 350 $\mu \mathrm{m}$.

Algumas irregularidades observadas são defeitos da cerâmica, tais como a porosidade e fissuras características da sua fabricação ou composição, conforme as imagens (a) e (b) da Fig. 2. Por outro lado, a partir da profundidade de $105 \mu \mathrm{m}$ pode ser notada uma influência maior da retificação na superfície da peça em ordem crescente. Esta análise é apoiada pelo aumento da severidade do processo de retificação, isto é, uma espessura de corte equivalente $\left(h_{e q}\right)$ maior. Notam-se efeitos mais significativos da retificação na superfície da peça quando a profundidade de corte de $210 \mu \mathrm{m}$ e $350 \mu \mathrm{m}$ foi utilizada. Infere-se que, com o aumento da taxa de remoção do material (aumentando o parâmetro $h_{e q}$ ), há um decréscimo na qualidade superficial, aumentando o número de fissuras nas imagens (e) e (f) da Fig. 2. Vale salientar que os riscos e as diferenças de cores nas imagens estão relacionados com as adjacências de vales formados. As cores quentes representam picos e as cores frias representam vales na superfície das peças.

A Fig. 3 apresenta a rugosidade das peças para as diferentes profundidades de corte usadas nos testes, os valores foram interpolados para facilitar a análise. Foi considerada a média dos quatros pontos medidos em cada peça, adicionando ao gráfico os intervalos de confiança. Observando a Figura 3, nota-se que o aumento da rugosidade é diretamente proporcional ao aumento da profundidade de corte, o que também é esperado devido à condição de usinagem ser mais agressiva, conforme a profundidade de corte aumenta. Foi calculado o coeficiente de determinação para os valores de rugosidade da Fig. 3, a partir de um ajuste linear e o valor foi de correlação foi de 0,870 , ou seja, a rugosidade se aproxima $87 \%$ do ajuste linear.

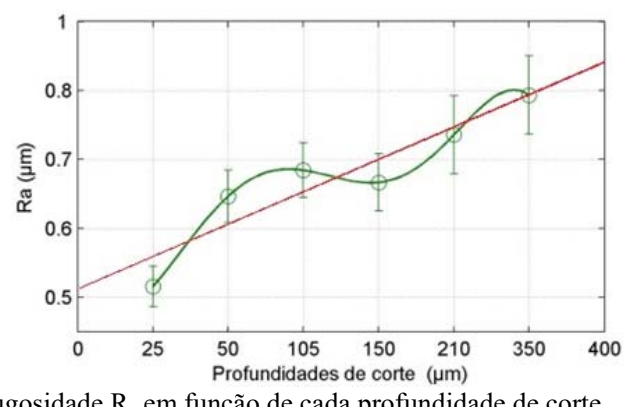

Figura 3. Rugosidade $\mathrm{R}_{\mathrm{a}}$ em função de cada profundidade de corte.

Na Fig. 3 podem notar-se alguns valores de desvio padrão mais elevados, principalmente nas ultimas condições de corte, que geralmente além da agressividade do processo, esses valores são usuais para medições da rugosidade, devido à variação dos picos e vales da superfície da peça. Em geral, os valores de rugosidade estão dentro de um faixa aceitável para o processo de retificação, visto que o maior valor observado na Fig. 3 está abaixo de $1 \mu \mathrm{m}$, sendo que o limite para processo, de acordo com Diniz [37] é de $1.6 \mu \mathrm{m}$.

\section{AVALIAÇÃO DO DESEMPENHO DO SINAL DE VIBRAÇÃO}

A seguir serão apresentados os resultados quanto processamento do sinal de vibração, com objetivo de avaliar seu desempenho no experimento. A Fig. 4 mostra o espectro do sinal de vibração obtido pela FFT e o PSD, o estudo do espectro de frequência do sinal de vibração teve como objetivo determinar uma correlação entre características desses sinais com as características das cerâmicas usinadas. Observa-se que a atividade vibracional nas frequências mais baixas se intensifica conforme a profundidade de corte é maior.

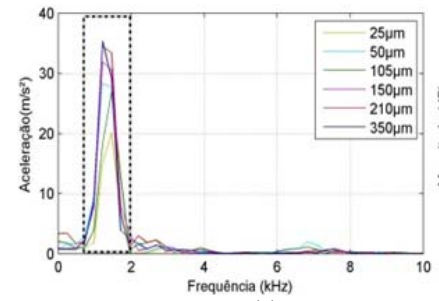

(a)

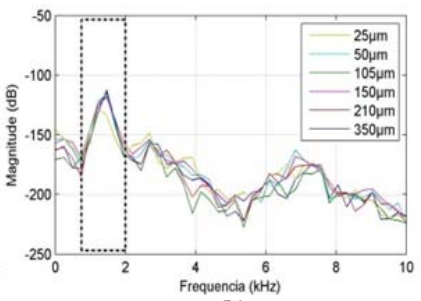

(b)
Figura 4. Seleção da melhor faixa de frquência no espectro de vibração; (a) FFT; (b) PSD.

Com base nessas observações, foi realizado o estudo em frequência do sinal de vibração utilizando janelas de Hanning em regiões de interesse do sinal, onde foi observada uma correlação mais próxima com as condições de corte. Observam-se baixas amplitudes e sobreposição nos sinais, nas faixas de frequência fora do trecho da passada, por exemplo, abaixo de $1 \mathrm{kHz}$ e acima $2 \mathrm{kHz}$. Por outro lado, na faixa de $800 \mathrm{~Hz}$ a $2 \mathrm{kHz}$ os sinais apresentam um comportamento interessante para estudo, por suas amplitudes serem maiores e as condições estarem bem definidas nesse trecho. Neste sentido foi aplicado no sinal puro de vibração um filtro digital nessa faixa encontrada na análise espectral, de acordo com o estudo da Fig. 5.

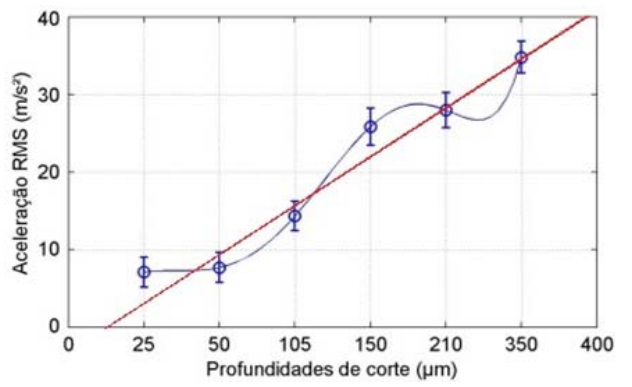

Figura 5. Sinal de vibração RMS filtrado em função das profundidades de corte.

Foi obtido o valor RMS e calculado valor médio juntamente aos intervalos de confiança no sinal de vibração. De acordo com a Fig. 5, na faixa de frequência selecionada, nota-se que a vibração aumenta conforme a profundidade de corte é maior 
principalmente após a profundidade de corte de $50 \mu \mathrm{m}$, ou seja, o comportamento segue uma tendência de aumento diretamente relacionada com o aumento da penetração da ferramenta na peça em cada passada de usinagem. O sinal de vibração RMS apresentou correlação com a rugosidade a partir do coeficiente de determinação calculado em função do ajuste linear. Para o sinal RMS de vibração o valor de correlação foi de 0,910 . Dessa forma, o sinal de vibração de aproxima $91 \%$ do ajuste linear.

Dessa forma, infere-se que existe uma atividade vibracional maior nas condições de usinagem mais agressivas. Por outro lado, foi observado anteriormente que também ocorreu um aumento da rugosidade das peças (Fig. 3) em função do aumento da profundidade de corte, assim como nas imagens de microscopia confocal, foram observados efeitos mais significativos da retificação nas condições de usinagem mais agressivas. Infere-se que o valor RMS da vibração (aceleração) pode ser usado para o monitoramento da rugosidade da peça para a retificação de cerâmica empregada neste trabalho, devido à correlação do mesmo com a rugosidade e qualidade superficial das peças. Com base na observação dos gráficos.

Na Fig. 6 foram obtidos os índices de danos RMSD e CCDM do sinal de vibração, a faixa de frequência de $800 \mathrm{~Hz}$ a $2 \mathrm{kHz}$, selecionada anteriormente, também foi a mais adequada para o cálculo desses índices, que neste estudo estão relacionados com a condição da superfície da peça. Os índices RMSD e CCDM foram normalizados para o cálculo, adotando o sinal de vibração da primeira peça como intacta e valor de amplitude igual a zero. O índice RMSD é um parâmetro muito preciso utilizado em analises para identificar um modelo diferente entre aqueles estudados, no caso deste trabalho foi em relação a amplitude do sinal para as diferentes profundidades utilizadas

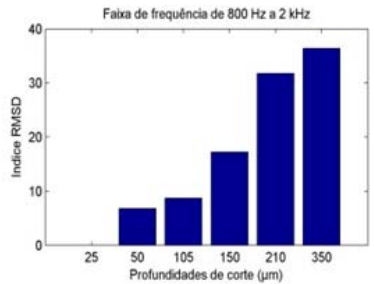

(a)

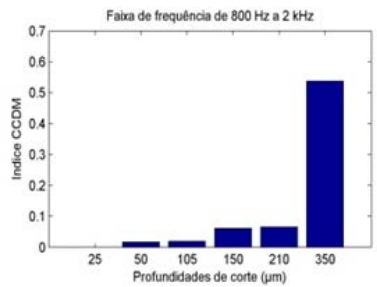

(b)
Figura 6. Índices de danos em função das profundidades de corte; (a) RMSD; (b) CCDM.

Pode observar na Fig. 6 que o RMSD apresenta de maneira satisfatória a correlação do sinal de vibração com a severidade do processo e, naturalmente com a rugosidade. Por outro lado, ja no índice CCDM, utilizado para encontrar correlação entre uma peça e outra em termos de frequência, com base na peça intacta, ou seja, a peça sem nenhuma evidencia de dano, que nesse caso, foi a peça usinada pela pronfudidade de $25 \mu \mathrm{m}$. Não foi obtido um padrão de comportamento que pudesse demonstrar um correlação com o sinal de vibração e as condiçoes das peças de cerâmicas no índice CCDM, pois o mesmo mostrou alteração repentina de comportamento em relação a ultima peça e as demais, conforme a Fig. 6 (b). Dessa forma, o índice CCDM não apresentou comportamento satisfatorio nesta análise.

\section{CONCLUSÃO}

Esta pesquisa apresentou um estudo das características do sinal de vibração relacionadas os efeitos da retificação plana de cerâmicas avançadas em diferentes profundidades de cortes. Com base nos resultados obtidos pode ser constatado que o sinal de vibração apresentou um relacionamento satisfatório com a condição das cerâmicas após a retificação, de acordo com a análise espectral e o estudo do sinal na faixa de frequência de $800 \mathrm{~Hz}$ a $2 \mathrm{kHz}$. Quanto maior a profundidade de corte, maior foi à rugosidade e o mesmo aconteceu com o sinal de vibração. Concluindo que o crescimento da rugosidade e vibração é diretamente proporcional ao aumento da severidade do processo de retificação. O índice de dano RMDS serviu de base para apoiar as conclusões desse trabalho sobre a relação do sinal de vibração com a integridade dos corpos de prova usinados. Os resultados do índice CCDM foram insatisfatórios para o presente estudo, pela inexistência de clareza no comportamento do sinal de vibração, concluindo que esse parâmetro não é adequado para o monitoramento do processo em questão.

\section{REFERÊNCIAS}

[1] S. Malkin and T. W. Hwang, "Grinding mechanisms for ceramics," CIRP Ann. Technol., vol. 45, no. 2, pp. 569-580, 1996.

[2] J. A. Kovach, P. J. Blau, S. Malkin, B. Srinivasan, S. Bandyopadhyay, and K. Ziegler, "A Feasibility Investigation of High Speed, Low Damage Girnding Process for Advanced Ceramics," 5th Int. Grind. Conf., vol. 1, no. SME.

[3] S. Malkin and J. E. Ritter, "Grinding Mechanism and Strength Degradation for Ceramics," ASME J. Eng. lnd, vol. 111, pp. 167173, 1989.

[4] E. Verlemann, "Technologies and Strategies for the Machining of Ceramic Components," Ceram. Monogr. No. 1.8.3.1, Varley Schmid GmbH., 1993.

[5] S. Agarwal and P. V. Rao, "A probabilistic approach to predict surface roughness in ceramic grinding," Int. J. Mach. Tools Manuf., vol. 45, no. 6, pp. 609-616, May 2005.

[6] M. S. Samhouri and B. W. Surgenor, "Surface roughness in grinding: on-line prediction with adaptive neuro fuzzy inference system," vol. 33, pp. 57-64, 2005.

[7] B. Dhanasekar, N. K. Mohan, B. Bhaduri, and B. Ramamoorthy, "Evaluation of surface roughness based on monochromatic speckle correlation using image processing," Precis. Eng., vol. 32, pp. $196-$ 206., 2008.

[8] D. J. Whitehouse, Handbook of Surface and Nanometrology, Second. 2010

[9] A. A. Fiocchi, "Ciência e tecnologia da manufatura de ultraprecisão de cerâmicas avançadas: Lapidorretificação Ud de superfícies planas de zircônia tetragonal policristalina estabilizada com ítria.," 2014.

[10] M. Elangovan, S. B. Devasenapati, N. R. Sakthivel, and K. I. Ramachandran, "Evaluation of expert system for condition monitoring of a single point cutting tool using principle component analysis and decision tree algorithm," Expert Syst. Appl., vol. 38, no. 4, pp. 4450-4459, 2011.

[11] C. H. R. Martins, P. R. Aguiar, A. Frech, and E. C. Bianchi, "Tool Condition Monitoring of Single-Point Dresser Using Acoustic Emission and Neural Networks Models," IEEE Trans. Instrum. Meas., vol. 63, no. 3, pp. 667-679, Mar. 2014.

[12] P. Sutowski and S. Plichta, "An investigation of the grinding wheel wear with the use of root-mean-square value of acoustic emission," Arch. Civ. Mech. Eng., vol. 6, no. 1, pp. 87-98, 2006.

[13] T. W. Liao, "Feature extraction and selection from acoustic emission signals with an application in grinding wheel condition monitoring," Eng. Appl. Artif. Intell., vol. 23, no. 1, pp. $74-84$.
Y. Zeng and E. Forssberg, "Monitoring Grinding Parameters By Vibration Signal Measurement - A Primary Aplication," vol. 7, no. 
R. Teti, K. Jemielniak, G. O’Donnell, and D. Dornfeld, "Advanced monitoring of machining operations," CIRP Ann. - Manuf. Technol., vol. 59, no. 2, pp. 717-739, Jan. 2010.

[16] F. W. SMITH, Principios de Ciências e Engenharia dos Materiais, $3^{\mathrm{a}}$ ed. Portugal, 1996.

[17] S. Y. LIN, Y. C. LIU, and C. . HUANG, "An investigation of surface grinding characteristics for titanium alloy with CBN wheel," Key Eng. Mater. Eng. Mater., vol. 364-366, pp. 237-242, 2008.

[18] S. Malkin and C. Guo, Grinding technology: theory and aplications of machining with abrasives, $2^{\mathrm{a}}$ ed. New York, 2008.

[19] E. C. Bianchi, P. R. Aguiar, E. J. Silva, J. Silva, and C. E. Fortulan, "Advanced ceramics: evaluation of the ground surface," Cerâmica, vol. 49, pp. 174-177, 2003.

[20] J. S. Kwak and M. K. HA, "Neural network approach for diagnosis of grinding operation by acoustic emission and power signals," $J$. Mater. Process. Technol., vol. 147 n1, pp. 65-71, 2004.

[21] W. J. and M. R.L, "Surface assessment of $\mathrm{CaF} 2$ deep-ultraviolet and vacuum-ultraviolet optical components by the quasi-Brewster angle technique," Appl Opt., vol. 45, pp. 5621-5628., 2006.

[22] B. MA, "Subsurface quality of polished $\mathrm{SiO} 2$ surface evaluated by quasi-Brewster angle technique," Optik (Stuttg)., vol. 122, pp. 4181422.

[23] J. Wang, S. Vankerkhove, and H. Schireiber, "Evaluation of coated and uncoated $\mathrm{CaF} 2$ optics by variable angle spectroscopic ellipsometry," Thin Solid Films, vol. 519, pp. 2881-2884, 2011.

[24] J. Neauport, "Imaging subsurface damage of grinded fused silica optics by confocal fluorescence microscopy," Opt. Express, vol. 17, pp. 3543-3554, 2009.

[25] H. Z. Wu, S. G. Roberts, and B. Derby, "Residual stress distributions around indentations and scratches in polycrystalline $\mathrm{Al} 2 \mathrm{O} 3$ and $\mathrm{Al} 2 \mathrm{O} 3 / \mathrm{SiC}$ nanocomposites measured using fluorescence probes," Acta Mater., vol. 56, pp. 140-149, 2008.

[26] J. H. Zhou, C. K. Pang, Z. W. Zhong, and F. L. Lewis, "Tool wear monitoring using acoustic emissions by dominant-feature identification," IEEE Trans. Instrum. Meas, vol. 60, pp. 547-559, 2011.

[27] A. Hassui and A. E. Diniz, "Correlating surface roughness and vibration on plunge cylindrical grinding of steel," Int. J. Mach. Tools Manuf., vol. 43, no. 8, pp. 855-862, Jun. 2003.

[28] J. W. Nilsson and S. A. Riedel, Circuitos Elétricos. 2003.

[29] A. Hassui, A. E. Diniz, J. F. G. Oliveira, J. Felipe Jr., and J. J. F. Gomes, "Experimental evaluation on grinding wheel wear through vibration and acoustic emission," Wear 217, pp. 7-14, 1998.

[30] C. DAVID and G. WARNECKC, "A correlation between cutting process and dynamics of the machine-tool-system in grinding.," Trans. NAMRI/ SME, vol. 24, pp. 51-56, 1996.

[31] C. Xu, Z. Liu, and W. Luo, "A frequency band energy analysis of vibration signals for tool condition monitoring," 2009 Int. Conf. Meas. Technol. Mechatronics Autom. ICMTMA 2009, vol. 1, pp. 385-388, 2009.

[32] E. O. Brigham, The fast fourier transform and its applications. Prentice-Hall International Editions, 1988.

[33] P. D. Welch, "The use of fast Fourier transform for the estimation of power spectra: a method based on time averaging over short, modified periodograms," IEEE Trans. Audio Electroacoust, 1967.

[34] T. Mathworks, Signal Processing Toolbox for Use with Matlab, User's Guide, V6 ed. 2002.

[35] J. Semmlow, "Biosignal and biomedical image processing MATLABbased applications," Marcel Dekker Inc., 2004.

[36] F. Sun, Z. Chaudhry, C. Linag, and C. A. Rogers, "Truss structure integrity identification using PZT sensor-actuator," J. Intell. Mater. Syst. Struct., vol. 6, pp. 134-139, 1995.

[37] A. E. Diniz, F. C. Marcondes, and N. L. Coppini, Tecnologia da usinagem dos materiais, $4^{\mathrm{a}}$ ed. Campinas, SP, 2003.

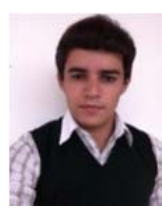

Pedro de Oliveira Conceição Junior Possui graduação em Tecnologia em Mecatrônica Industrial, pela Faculdade de Tecnologia do Estado de São Paulo, (2013). Atualmente é doutorando e concluiu mestrando em Engenharia Elétrica pela Universidade Estadual Paulista "Júlio de Mesquita Filho", Campus de Bauru SP. Atualmente é professor na Faculdade Anhanguera de Bauru SP. Atua na linha pesquisa Mecatrônica, principalmente com os temas de: Monitoramento de Processos de Manufatura com investigação por Fusão de Sensores, aplicação de ferramentas de Aquisição de Dados, Processamento Digital de Sinais e Inteligência Computacional em processos especialmente voltados para a usinagem por abrasão.

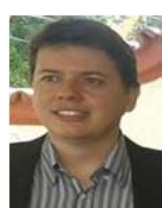

Marcelo Marchi Possui mestrado e doutorado em Engenharia Mecânica pela Universidade Estadual Paulista (UNESP) Campus Bauru-SP, Especialista em Desenvolvimento de Sistemas para Internet pela Universidade de Ribeirão Preto (UNAERP). Graduado em Computação (Licenciatura Plena) pelo Centro Universitário Claretiano. Atualmente é professor do Centro Estadual de Educação Tecnológica Paula Souza - SP. Tem experiência na área de Ciência da Computação, com ênfase em Microeletrônica.

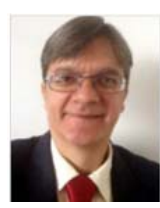

Paulo Roberto de Aguiar é Professor Titular do Departamento de Engenharia Elétrica da UNESP Bauru. Realizou programa de Pós-Doutorado na University of Connecticut, USA na área de Processos de Fabricação. Mestre em Engenharia Elétrica pela USP São Carlos e doutor em Engenharia Elétrica pela USP São Paulo. Livre-Docência pela UNESP Bauru nas áreas de Sistemas de Controle e Processos de Fabricação. Formado em Engenharia Elétrica pela Universidade de Bauru (atual FEB-Unesp). Atualmente é coordenador do Program a de PósGraduação em Engenharia Elétrica da Faculdade de Engenharia de Bauru, UNESP.

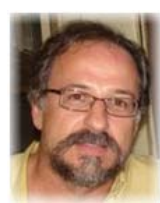

Eduardo Carlos Bianchi é Professor Titular do Departamento de Engenharia Mecânica UNESP Bauru. Mestre e doutor em Engenharia Mecânica pela USP São Carlos. Livre Docente pela UNESP Bauru na área de Engenharia Mecânica. Formado em Engenharia Mecânica e Engenharia de Segurança do Trabalho pela UNESP Bauru. por Abrasão. Atualmente coordena o Grupo de Pesquisas em Usinagem

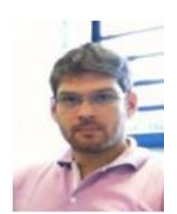

Thiago Valle França Possui graduação em Engenharia Mecânica pela Universidade Estadual Paulista (2002), mestrado em Engenharia Industrial pela Universidade Estadual Paulista (2005), doutorado em Engenharia de Produção pela Universidade de São Paulo (2009) com estágio na TRW Fuji Valve (EUA) e pós-doutorado na Georgia Institute of Technology, Atlanta-GA, EUA. Tem experiência na área de Engenharia Mecânica, com ênfase em Processos de Fabricação, atuando principalmente nos seguintes temas: desgaste abrasivo, retificação, otimização de processos de fabricação, mínima quantidade de lubrificação, altas velocidade de retificação, vibração em processos de fabricação, monitoramento e retificação assistida à laser. Atualmente é Professor Assistente Doutor do Departamento de Engenharia Mecânica da Universidade Estadual Paulista, Câmpus Bauru - UNESP. 